\title{
Modelling the Force Action of a Liquid on the Shutter of a Measuring Transducer
}

\author{
Vitaly Golubovsky ${ }^{1}$, Vladimir Konovalov ${ }^{1 *}$ and Marina Doncova $^{1}$ \\ ${ }^{1}$ Department of Machine Building Technology, Penza State Technological University, 440039, 1A/11 \\ Baydukova Travel / Gagarina Street, Penza, Russia
}

\begin{abstract}
The article describes the original design of the measuring transducer of angular velocity of the type "flapper-nozzle ". The aim of the study is mathematical modeling in the MathCAD program of the force action of liquid jets exiting nozzles, since this significantly affects the characteristics of the converter. The calculated values of the acting forces are compared by several known methods and their results are compared with experimental data. For the proposed design of the measuring transducer, greater convergence of the theoretical and experimental values of the power characteristic in the linear section is provided by the expressions for determining the force effect of two jets on the damper, defined as the hydrodynamic effect of the liquid jet on the obstacle. Given the real differences in the design of the converter from theoretical models, it is required to apply correction factors. As pressure increases, the coefficient increases from 1 to 1.24-1.30 depending on the pressure supplied to the measuring transducer and the version of expression used to calculate it. When using the expression for the approximate calculation of the acting forces in the converter, the correction factor is much higher and varies from 1.9 at a supply pressure of $0.5 \mathrm{MPa}$ to 1.74 at a supply pressure of 1.5-2.0 MPa. The practical use of this calculation option is limited to the linear zone of pressure dependence in the working chambers of the converter on the gap between the nozzles and the shutter.
\end{abstract}

\section{Introduction}

Currently, a large number of technological equipment $[1,2,3]$ having hydraulic drives $[4$, $5]$ is equipped with automatic control systems. The analysis showed that for hydraulic automatic control systems it is advisable to use measuring transducers based on a hydraulic amplifier of the "flapper-nozzle" type [6, 7, 8].

For hydraulic drives of rotational motion, angular-speed transducers, which determine the quality of transients in the automatic control system should be used. Such a measuring transducer is designed to measure and control the angular speed of objects in automatic control systems of hydraulic drives of industrial equipment.

Figure 1 shows a structural diagram of a measuring angular-speed transducer of the "flapper-nozzle" type [9]. The angular-speed transducer is located in the rotor 15, and

*Corresponding author: konovalov-penza@rambler.ru 
consists of a housing 1 , stops 20 and 21 , covers 16 and 17 with screws 18 and 19 , fixed with nuts 22 and 23. The sensing element consists of nozzles 2 and 3, constant throttles 5 and 6 , an inertia flapper 4 , as well as of centering springs 12 and 13 . The measuring angular-speed transducer operates as follows.

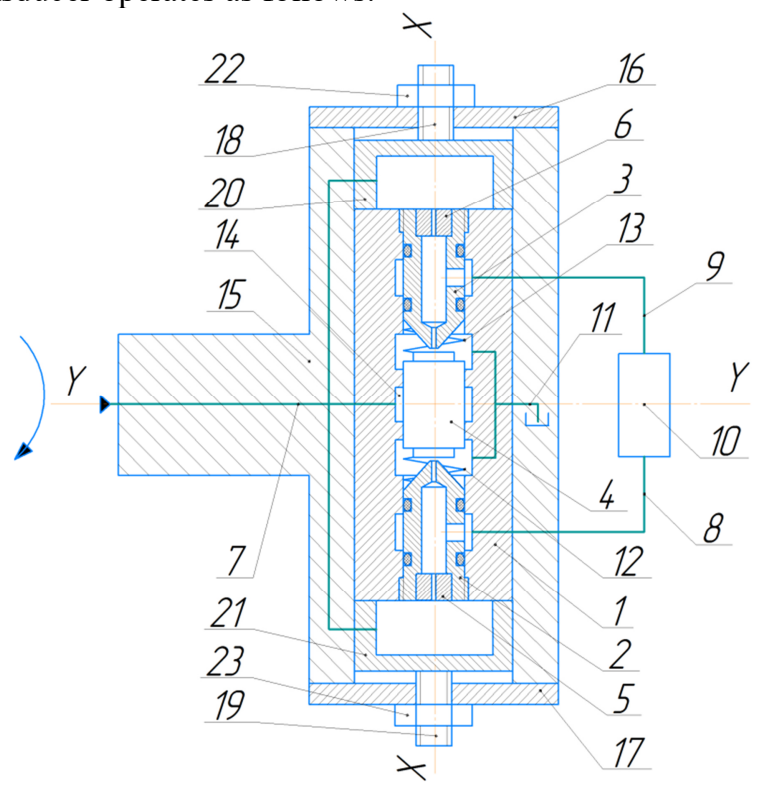

Fig. 1. A structural diagram of a measuring angular-speed transducer: 1 - housing; 2,3 - nozzles; 4 inertia flapper; 5, 6- constant throttles; 7 -hydraulic fluid line; 8, 9 - hydraulic control signal lines; 10 - actuating element; 11 - hydraulic line for draining liquid into a container; 12,13 - springs; 14 radial hydraulic support; 15 - measuring transducer rotor; 16, 17 - covers; 18, 19 - adjusting screws; 20, 21 - stops; 22, 23 - nuts; X-X - transducer sensitivity axis; Y-Y - transducer axis of rotation.

If the rotor 15 does not rotate, then there are no inertia forces in the direction of the sensitivity axis. The working fluid from the supply line 7 flows through the constant throttles 5 and 6 into the measuring chambers of the nozzles 2 and 3, and then, having passed the resistance in the form of gaps between the ends of the nozzles and the flapper 4, is discharged into the tank via the line 11. The inertia flapper 4 is in equilibrium, occupying a symmetrical position in the center of the housing, under the action of the forces of the centering springs 12 and 13 . This leads to the creation of the same resistance to the outflow of the working fluid from the nozzles and equal pressure in the measuring chambers $p_{1}=p_{2}$. The working fluid under pressure is supplied to the central borehole 14 of the transducer housing, creates a centering force evenly distributed over the cylindrical surface, holding the flapper 4 on the sensitivity axis. The fluid passing from the central borehole into the drain cavities of the transducer and then to the line 11 through the radial gaps between the housing and the flapper prevents dry friction.

When the rotor 15 of the transducer rotates at a certain angular speed, a centrifugal inertia force arises along the sensitivity axis (for example, up). Under the action of this force, the flapper 4 moves up and changes the hydraulic resistance of the nozzles 2 and 3. The resistance to the flow of oil from the nozzle 2 decreases, and from the nozzle 3 it increases, which leads to a corresponding change in the pressure in the measuring chambers $p_{1}<p_{2}$. The resulting pressure difference $\left(\Delta p_{m t}=p_{2}-p_{1}\right)$ is used as a control signal at the inputs of the actuating element 10 , for example, a throttling spool valve. 


\section{Research methods}

The research methodology provided a comparative analysis of the known expressions for determining two-sided force effect of liquid jets on the flapper of the measuring transducer of the "flapper-nozzle" type in order to substantiate the most adequate expression, as applied to a particular design, with the refinement of the expressions of the correction factors.

The results of modelling the force effect obtained by numerical modelling in the MathCAD program, and their verification was carried out by comparison with experimental data.

The values of the impact force of the jet were determined at given values of the supply pressure of the transducer, as well as at a specific gap between the nozzles and the flapper. The experimental data for comparison with theoretical expressions were determined as the difference between the values of the forces of action of the reciprocal jets acting on the flapper with a coordinated difference in the distances between the nozzles and the flapper. The shape and surface area of the working profile of the flapper from the side of both nozzles was the same. The distance between the nozzles was always constant, as was the total gap between them and the flapper, which was $0.5 \mathrm{~mm}$. The gap between the specific nozzle and the flapper was determined by the position of the movable flapper and was an independent research factor. The gap between the first nozzle and the flapper was $h_{1}=(0.5$; $0.4 ; 0.3 ; 0.25 ; 0.2 ; 0.1$ and $0 \mathrm{~mm})$. At the same time, for the second nozzle and the flapper, the gap was $\mathrm{h}_{2}=(0 ; 0.1 ; 0.2 ; 0.25 ; 0.3 ; 0.4$ and $0.5 \mathrm{~mm})$. The transducer supply pressure was $0.5 ; 1.0 ; 1.5$ and $2.0 \mathrm{MPa}$.

Figure 2 shows a section of the working part of the adjustable throttle nozzle-flapper. The flow of the working fluid, falling from the nozzle opening 2 into the working gap, gradually narrows. Therefore, in the center of the nozzle opening, a pressure cone 3 is formed, the base of which lies on the surface of the flapper 1 . In the zone bounded by this cone, the average fluid velocity is zero, therefore, the pressure in this region is equal to the pressure $p_{1}$ on the surface of the central part of the flapper.

We consider the fluid motion in a relatively small gap $h_{i 1}$ (to the left of the axis in Figure 2). The fluid flow, falling into the gap between the nozzle and the flapper, breaks off from the sharp edge of the nozzle formed by the hole and the plane of the end face.

At some distance, the extreme boundaries of this flow do not touch the end of the nozzle, and then adhere to it. In zone 4, where the flow breaks away from the end of the nozzle, there is the greatest narrowing of the flow, which leads to an increase in its speed. Therefore, the pressure decreases and in the zone of narrowing of the flow, it can take negative values. This flow regime is continuous, that is, the flow actually fails to tear itself away from the end of the nozzle.

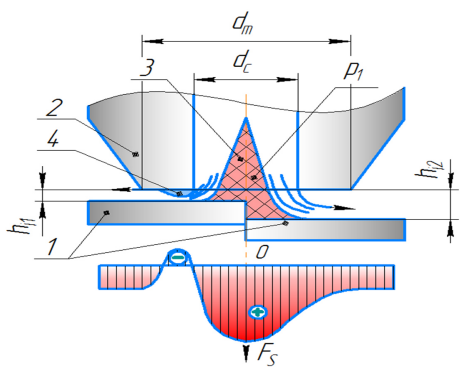

Fig. 2. Scheme of fluid flow in the adjustable part of the flapper-nozzle throttle: 1 - flapper; 2 nozzle; 3 - pressure cone; 4 - zone of narrowing of the flow. 
At large values of the gap $h_{i 2}$ (to the right of the axis in Figure 2), the fluid flow, breaking away from the edge formed by the nozzle hole and the plane of its end face, moves without adhering to the end face of the nozzle. This flow regime is tear-off [10].

The out flowing jet acts on the flapper with a force $F_{S}$ equal in magnitude to the sum of three forces: a) the force caused by a change in the momentum of the mass of the working fluid flowing from the nozzle; b) the force caused by the pressure of the working fluid on the surface of the outlet section of the nozzle; c) the force due to the pressure of the working fluid in the gap between the end of the nozzle and the flapper $[11,12]$ :

$$
F_{S}=\left[8 \pi \mu_{n f}^{2} h^{2}+\frac{\pi}{6}\left(\frac{d_{m}^{2}}{2}+\frac{d_{c}^{3}}{d_{m}}\right)\left(1-\frac{16 \mu_{c 3}^{2} h^{2}}{\mu_{c}^{2} d_{c}^{2}}\right)\right] p_{1},
$$

where $\mu_{n f}$ - flapper-nozzle discharge coefficient; $\mu_{c}$ - nozzle discharge coefficient, as a constant throttle; $h$-gap between the nozzle and the flapper; $d_{m}$ - nozzle end diameter; $d_{c}$ - nozzle hole diameter; $p_{1}$ - inter-throttle chamber pressure.

The formula (1) can be represented as:

where

$$
F_{S}=\alpha_{1} p_{1}+\left(\alpha_{2}-\alpha_{3}\right) Q_{m t}^{2}
$$

$$
\left.\begin{array}{l}
\alpha_{1}=\frac{\pi}{6}\left(\frac{d_{m}^{2}}{2}+\frac{d_{c}^{3}}{d_{m}}\right) \\
\alpha_{2}=\frac{4 \rho}{\pi d_{c}^{2}} \\
\alpha_{3}=\frac{4 \rho}{3 \pi d_{c}^{4} \mu_{c}^{2}}\left(\frac{d_{m}^{2}}{2}+\frac{d_{c}^{3}}{d_{m}}\right)
\end{array}\right\},
$$

where $d_{m}$ - nozzle end diameter; $d_{c}$ - nozzle hole diameter; $\rho$ - working fluid density; $\mu_{c}$ - nozzle discharge coefficient.

Figure 3 shows a schematic diagram of a measuring transducer of angular speed.

The difference in force on the flapper will be equal to:

$$
\Delta F_{S}=F_{S 1}-F_{S 2}=\alpha_{1}\left(p_{1}-p_{2}\right)+\left(\alpha_{3}-\alpha_{2}\right)\left(Q_{m t 1}^{2}-Q_{m t 2}^{2}\right) \text {, }
$$

where $F_{S 1}$ and $F_{S 2}$ - forces on the flapper from nozzles 1 and 2 (see Figure 3); $Q_{m t 1}$ and $Q_{m t 2}$ - fluid flow through nozzles 1 and 2 .

For an approximate calculation of the force effect of the flow on the flapper in practice, one usually uses a formula that is applicable mainly to nozzles with a ratio of diameters of $d_{m} / d_{c}=1.2[10]$ :

$$
F_{S}=\frac{\pi d_{c}^{2}}{4} \Delta p_{m t}
$$

where $\Delta p_{m t}=p_{1}-p_{2}$ - differential pressure in the diagonal of the hydraulic bridge.

The hydrodynamic force of the jet on the obstacle is determined by the formula:

$$
F_{S}=\rho \vartheta Q=\rho S \vartheta^{2}
$$

where $\rho$-fluid density; $\vartheta$ - fluid flow rate through the nozzle; $Q$ - fluid flow through the nozzle; $S$ - nozzle hole area.

Then the resulting force of the jets on the flapper is determined as 


$$
\Delta F_{S}=\rho \frac{\pi d_{c}^{2}}{4}\left(\left(\frac{4 \mu_{n f}{ }_{2} Q_{4}}{\pi d_{c}^{2}}\right)^{2}-\left(\frac{4 \mu_{n f 1} Q_{3}}{\pi d_{c}^{2}}\right)^{2}\right),
$$

where $d_{c}$ - nozzle hole diameter (in this transducer, nozzle hole diameters are equal); $\mu_{n f 1}$ and $\mu_{n f} 2$ - discharge coefficient of nozzles 1 and 2; $Q_{3}$ and $Q_{4}$ - fluid flow through nozzles 1 and 2 .

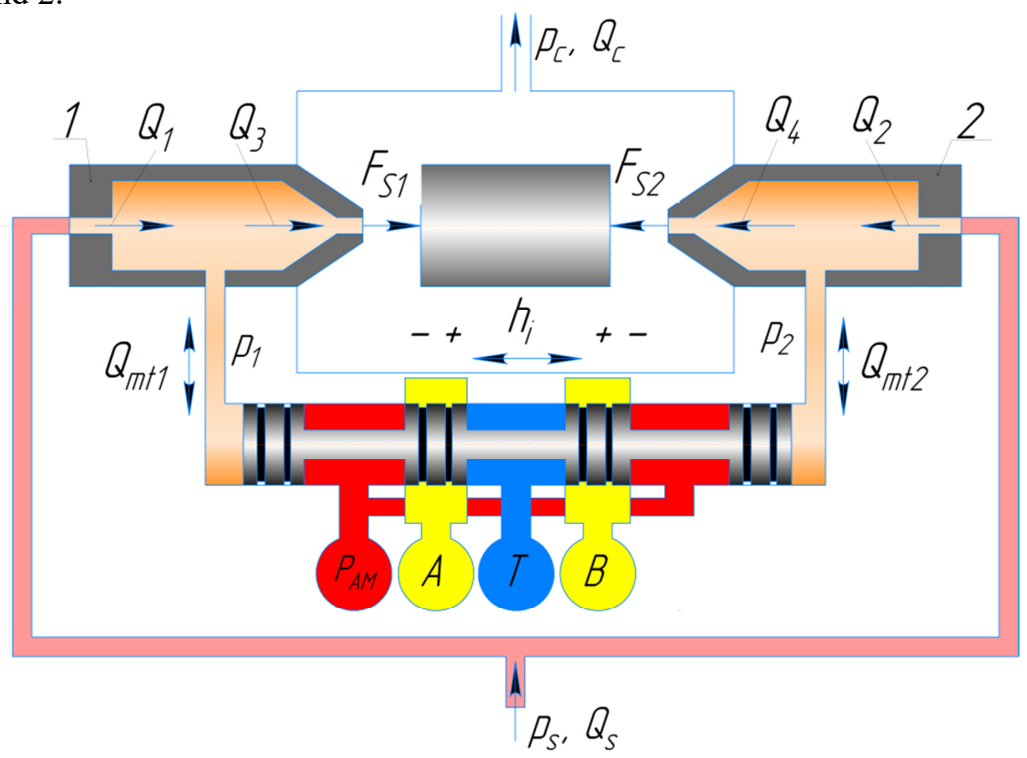

Fig. 3. Schematic diagram of the measuring transducer.

\section{Research result}

The modelling results obtained for the fluid flow rate and supply pressure of the transducer based on previously obtained experimental data are presented in Figure 4. The numerical values of the source data for all three calculation options correspond to each other. In Figure 4.(a) - a dependence based on the formula 4. In Figure 4.(b) - a dependence based on the formula 5. In Figure 4.(c) - a dependence based on the formula 7.

For better visualization, comparisons of different expressions for calculating the difference between the force effects of the jets on the flapper between them, we bring them together in one graph and additionally indicate the difference in forces (total effect) of the jets on the flapper according to experimental data (point values in Figure 5). In this case, Figure 5.(a) gives a graphical dependence without introducing a correction factor. Figure 5.(b) shows a graphical dependence of the total effect of the jet forces, taking into account the correction coefficient, expressed as a cofactor of the indicator to match the experimental dependence.

If the area of the annular gap between the nozzle and the flapper is less than the area of the nozzle hole, a linear section of the dependence of the force of the jet on the flapper on the gap is observed. These sections are presented in Figure 5 in the interval of the flapper position from one of the nozzles in the interval ho $=0.0001-0.0004 \mathrm{~m}$.

As a result of the regression processing of the values of the correction factors, expressions were obtained that describe the dependence of the total force action of the jets depending on the supply pressure supplied to the transducer. 

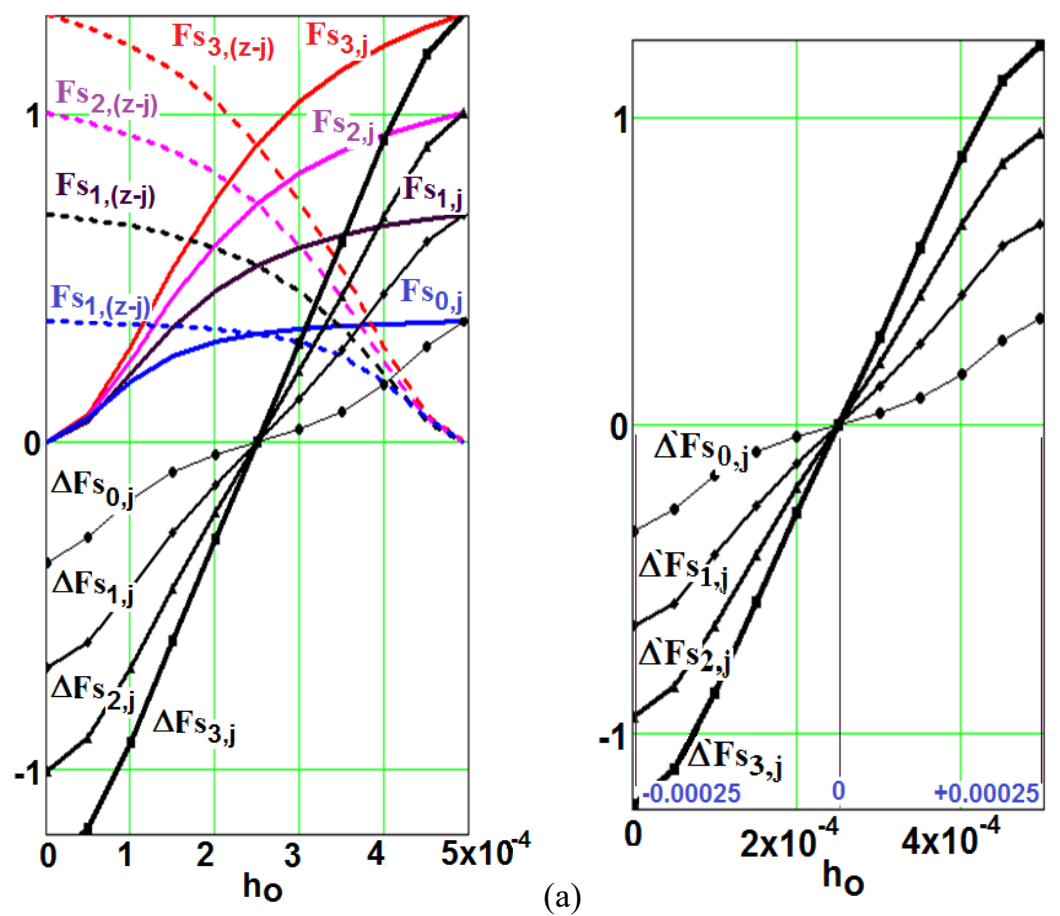

(a)

(b)

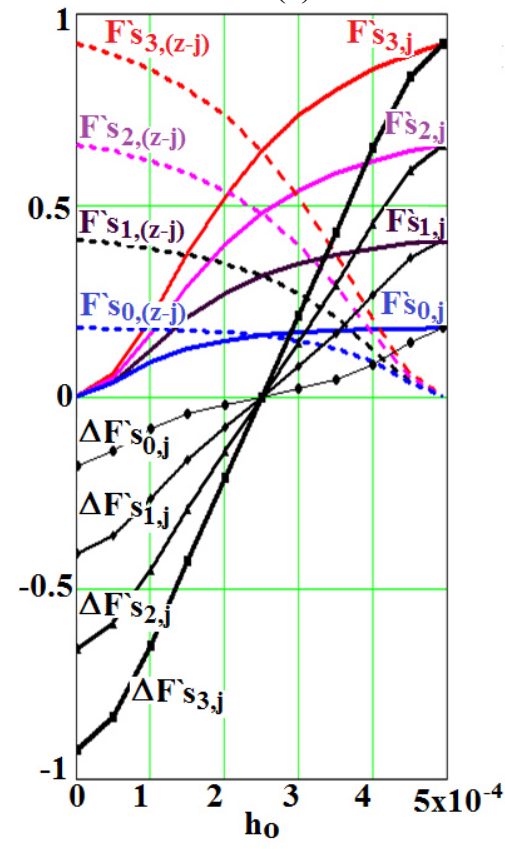

(c)

Fig. 4. The calculated values of the effective forces of the liquid jet FS and the difference in the action (resulting value) of the forces $\triangle \mathrm{FS}$ of the liquid jets acting through both nozzles for different pressure of the liquid at the inlet of the transducer $(\mathrm{i}=0-0.5 \mathrm{MPa} ; \mathrm{i}=1-1.0 \mathrm{MPa} ; \mathrm{i}=2-1.5 \mathrm{MPa} ; \mathrm{i}=3$ $-2.0 \mathrm{MPa}$ ), taking into account the gap $\mathrm{h}$ between the nozzle and the flapper, according to the calculation formulas: (a) - f.4; (b) - f.5; (c) - f.7.

The smallest values of the correction factor for the expression described by the recommended expressions for hydraulic amplifiers (Figure 6): 


$$
\mathrm{K}_{1}=0.847429+0.275 \cdot \mathrm{P}^{0.518386} .
$$

F-test $=0.99948$ and Pearson correlation coefficient $\mathrm{R}=0.999591$ indicate the adequacy of the resulting expression.

With an increase in the supply pressure of the transducer in the gap between the nozzle and the flapper, the flow turbulence increases. Due to this and the impossibility of a turbulent fluid flow to leave the chamber between the nozzle and the flapper instantly, an additional dynamic pressure arises, which is taken into account by the above coefficient. Depending on the value of the supply pressure, the coefficient increases from 1 to 1.24 with an increase in the supply pressure of the transducer to $2.0 \mathrm{MPa}$.

The abovementioned phenomenon and the actual direction of fluid flow from the nozzle opening upon impact with the flapper, as well as the subsequent movement of the reflected jet from the flapper to the flat end of the nozzle and further reflection of the jet from the nozzle end to the flapper and from the flapper to the nozzle cone leads to a partially reactive movement (Figure 2), which affects the magnitude of the force action of the liquid on the valve. This takes into account the correction factor for calculation based on the force of action of the jet on the obstacle according to the expression (f.7):

$$
\mathrm{K}_{3}=0.874353+0.2732 \cdot \mathrm{P}^{0.65278} \text {. }
$$

F-test $=0.997044$ and Pearson correlation coefficient $\mathrm{R}=0.997681$ indicate the adequacy of the resulting expression.

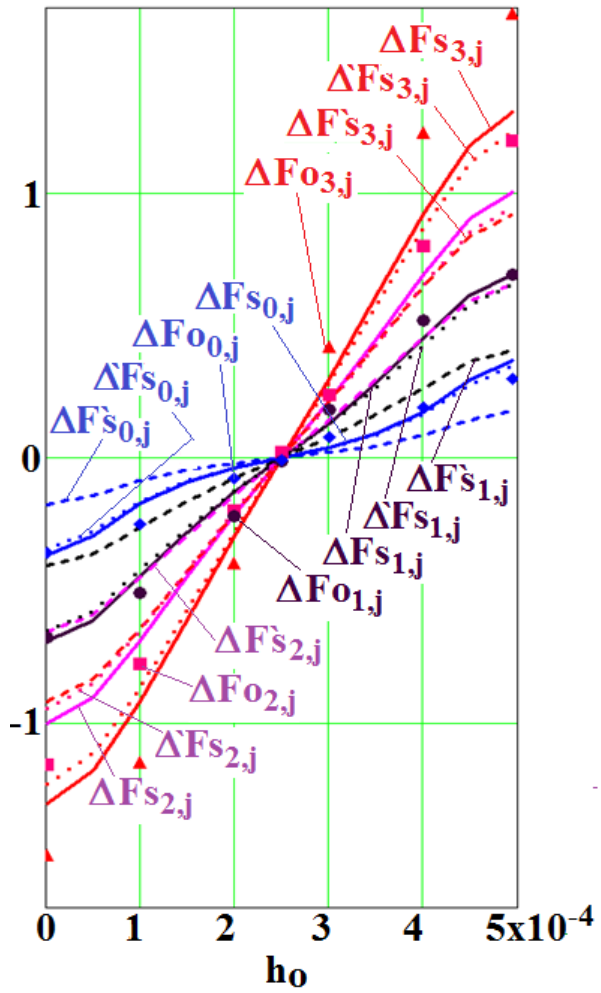

(a)

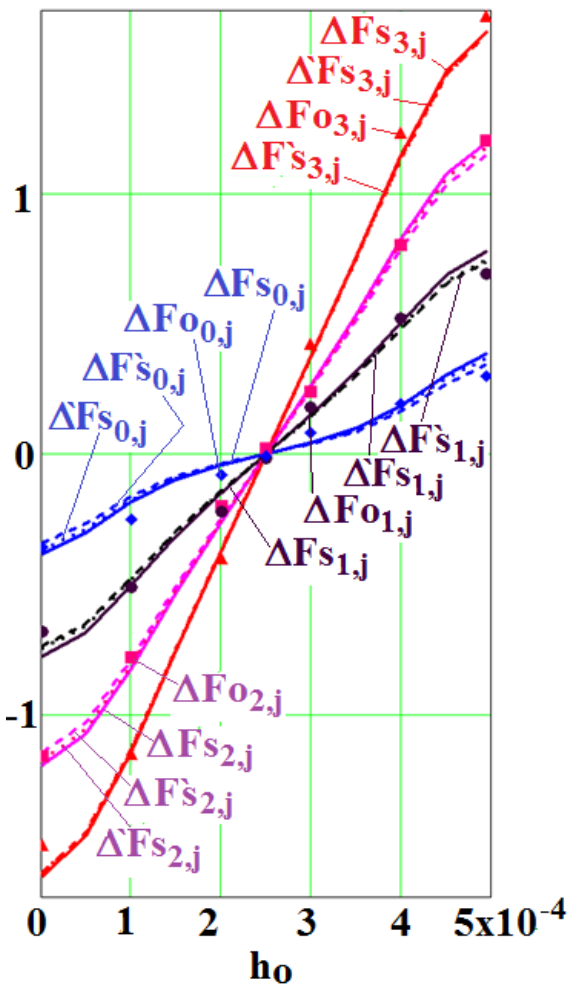

(b)

Fig. 5. The calculated values of the difference in the action (total value) of the forces of the liquid jets $\Delta \mathrm{F}_{\mathrm{S}}$ acting through both nozzles under different supply pressure of the transducer $(\mathrm{i}=0-0.5 \mathrm{MPa} ; \mathrm{i}=1$ $-1.0 \mathrm{MPa} ; \mathrm{i}=2-1.5 \mathrm{MPa} ; \mathrm{i}=3-2.0 \mathrm{MPa}$ ) taking into account the gap $\mathrm{h}_{\mathrm{oj}}$ between the nozzle and the flapper according to the calculated expressions: f.4 $-\Delta \mathrm{F}_{\mathrm{S}}$; f.5 - $\Delta \mathrm{F}_{\mathrm{S}}{ }_{\mathrm{S}}$; f.7- $\Delta{ }^{\prime} \mathrm{F}_{\mathrm{S}}$; experimental data $\Delta \mathrm{F}_{0}$; (a) - without the introduction of a correction factor; (b) - with a correction factor as an indicator factor. 
With an increase in the supply pressure of the measuring transducer, the indicated coefficient increases from 1.05 at a supply pressure of $0.5 \mathrm{MPa}$ to 1.3 at a supply pressure of 2.0 MPa.

The highest numerical values of the correction coefficient are given by the formula for the approximate calculation of the acting forces on the flapper of the transducer. It is described by the expression (Figure 6):

$$
\mathrm{K}_{2}=1.6568544+0.136 \cdot \mathrm{P}^{-0.84283} \text {. }
$$

F-test $=0.98922$ and Pearson correlation coefficient $\mathrm{R}=0.991569$ indicate the adequacy of the resulting expression.

The correction factor in this case changes from 1.9 at a supply pressure of $0.5 \mathrm{MPa}$ and stabilizes to 1.74 with an increase in supply pressure from 1.5 to $2.0 \mathrm{MPa}$.
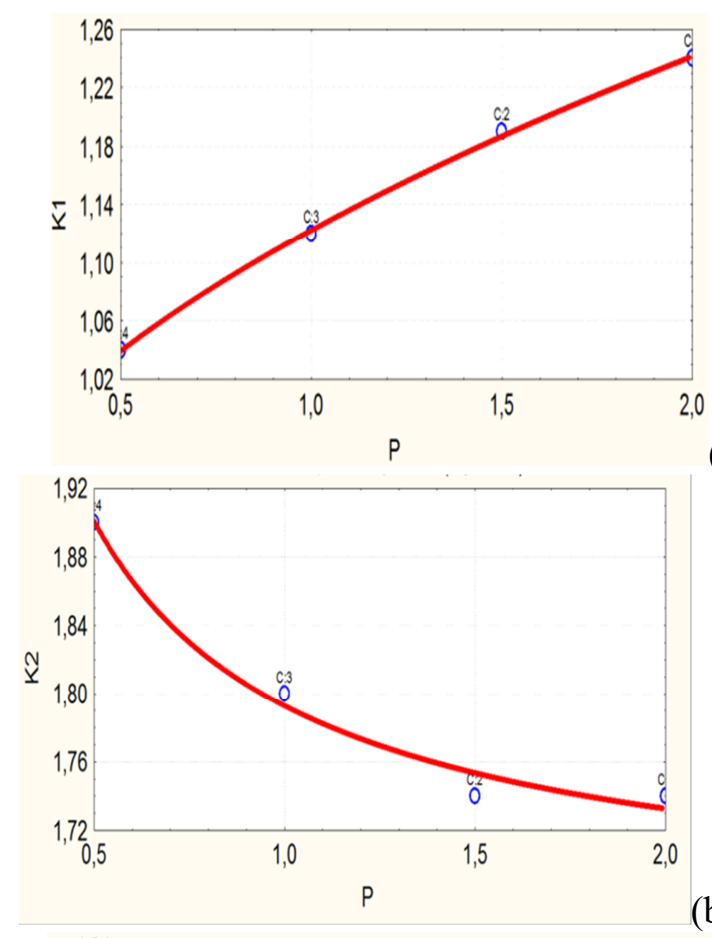

(a)

(b)

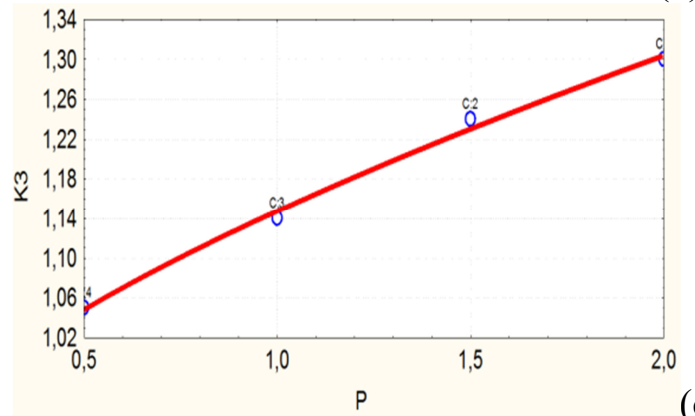

(c)

Fig. 6. Change in the numerical values of the correction factors depending on the supply pressure of the transducer: a) $\mathrm{K} 1$ - correction factor for calculating the force effect according to the formula 4; b) $\mathrm{K} 2$ - correction factor for calculating the force effect according to the formula 5; c) $\mathrm{K} 3$ correction factor for calculating the force effect according to the formula 7. 


\section{The conclusion}

The performed modelling of changes in the resulting force effect of two jets on the flapper of the measuring angular speed transducer of the "flapper-nozzle" type made it possible to establish the expressions that most accurately describe the power characteristic of the transducer. For the proposed design of the measuring transducer, greater convergence of the theoretical and experimental values of the power characteristic in the linear section is provided by the expressions for determining the force effect of two jets on the flapper, defined as the hydrodynamic effect of the liquid jet on the obstacle. Given the real differences in the design of the measuring transducer from theoretical models, it is required to apply correction factors. As pressure increases, the coefficient increases from 1 to 1.24 1.30 , depending on the supply pressure of the transducer and the version of expression used to calculate it.

When using the expression for the approximate calculation of the acting forces on the flapper, the correction factor changes from 1.9 at a supply pressure of $0.5 \mathrm{MPa}$ to 1.74 when the pressure changes from 1.5 $\mathrm{MPa}$ to $2.0 \mathrm{MPa}$. The practical use of this calculation option is limited to the linear zone of pressure dependence in the working chambers of the transducer on the gap between the nozzles and the flapper.

\section{References}

1. I. Kadyrov, B. Turusbekov, Development of an Automatic System of Stabilization Thrust Force at the Turning Operations and its Mathematical Model, Proceedings 2019 1st International Conference on Control Systems, Mathematical Modelling, Automation and Energy Efficiency, SUMMA-2019, 8947485, 255-257, (2019). DOI: 10.1109/SUMMA48161.2019.8947485.

2. V. Sokolov, O. Krol, Y. Baturin, Dynamics Research and Automatic Control of Technological Equipment with Electrohydraulic Drive. Proceedings - 2019 International Russian Automation Conference, RusAutoCon-2019, 8867652. (2019) DOI: 10.1109/RUSAUTOCON.2019.8867652.

3. E. Karabegović, E. Šemić, S. Isić. Automatic Control of Tube Hydroforming Process in Experimental Conditions, Lecture Notes in Networks and Systems, 42, 101-106, (2019). DOI: 10.1007/978-3-319-90893-9_11.

4. V. Sokolov, O. Krol, O. Stepanova, Automatic control system for electrohydraulic drive of production equipment, International Russian Automation Conference, RusAutoCon-2018, 8501609. (2018). DOI: 10.1109/RUSAUTOCON.2018.8501609

5. W. Armbruster, G. Schmid Hydraulic copyer control in machine tools, Tz prakt. Metallbearb, 73, 19-22, (2017). https://www.scopus.com/inward/record.uri?eid=2-s2.00013727278\&partnerID=40\&md5=934ada7522b1 f5f79d0ff8957597cb39.

6. Y. Medvedev, V. Kuznetsov, New control and executive devices for multimotor electrohydraulic drives, Russian Engineering Research 31(9), 828, (2011). DOI: 10.3103/S1068798X11090206

7. Y. Medvedev, V. Kuznetsov, Dynamics of a Multimotor Electrohydraulic Drive in an Automatic Control System, Russian Engineering Research, 31(6), 527, (2011). DOI: 10.3103/S1068798X11060165

8. A. Bazhenov, E. Levichev, The dynamics of hydraulic distributors of the nozzle-gate and jet-tube types, Engineering Bulletin, 7, 17, (1988). https://www.scopus.com/inward/record.uri?eid=2-s2.00023701727\&partnerID=40\&md5=16b65b368baa13889ee0022be772c290 
9. N. A. Simanin, V. V. Golubovsky, FIPS information Bulletin, 13, (2018)

10. Yu. I. Chuprakov, Hydraulic drive and means of hydroautomatics, Moscow. Mechanical Engineering, 232, (1979)

11. I. M. Krassov, L. I. Radovsky, B. G. Turbin, Approximate determination of the jet reaction in a hydraulic flapper-nozzle power assist, Automatics and telemechanics, 11, $1536-1538,(1960)$

12. I. M. Krassov, L. I. Radovsky, B. G. Turbin, Power effect of a jet in a hydraulic booster nozzle-flap, Automatics and telemechanics, 12, 1635 - 1651, (1959) 